Furthermore $3 \mathrm{mM} \mathrm{Pi}$ treatment increased glucose uptake (98\%, p<0.05) and Glut-1 mRNA expression (1.47 fold, $\mathrm{p}<0.001)$. Glycolysis converts glucose to pyruvate which is subsequently converted to either (i) acetyl-CoA by the pyruvate dehydrogenase complex $(\mathrm{PDH})$ or (ii) lactate by lactate dehydrogenase (LDH). Notably, decreased VSMC calcification was observed in cells treated with sodium dicholoroacetate, an inducer of $\mathrm{PDH}$ activity $(1 \mathrm{mM} ; 40 \% ; \mathrm{p}<0.01)$ and citric acid, synthesised in the mitochondria from acetyl CoA (1 mM; 72\%, p <0.001). Treatment with the LDH inhibitor sodium oxamate $(20 \mathrm{mM})$ or sodium lactate $(50 \mathrm{mM})$ to induce pyruvate production also inhibited VSMC calcification $(68 \%$ and $53 \%$ respectively, $\mathrm{p}<0.05)$. Activation of the Wnt pathway - an established regulator of Warburg metabolism using the selective GSK3 inhibitor CHIR99021 (1 nM) significantly increased VSMC calcification (417\%, p<0.001). However, co-treatment with sodium oxamate $(20 \mathrm{mM})$ significantly blunted the pro-calcification effect of CHIR99021 (69\%, $\mathrm{p}<0.01)$.

Conclusion Together these data suggest that arterial calcification requires glucose metabolism through a mechanism involving Wnt signalling. Interruption of the glycolysis pathway may therefore represent a novel therapeutic target for clinical intervention.

\section{VASCULAR ENDOTHELIAL GROWTH FACTOR RECEPTOR INHIBITION INDUCES VASCULAR DYSFUNCTION VIA REDOX-SENSITIVE PROCESSES}

${ }^{1}$ Karla Bianca Neves*, ${ }^{1}$ Francisco J Rios, ${ }^{1}$ Augusto C Montezano, 'Lucas Van Der Mey, ${ }^{2}$ Carmine Savoia, ${ }^{1}$ Rhian Touyz. ${ }^{1}$ University of Glasgow; ${ }^{2}$ University of Roma

10.1136/heartjnl-2017-311726.222

Vascular endothelial growth factor (VEGF)/VEGF receptor (VEGFR) inhibitors, used as anti-angiogenic drugs to treat cancer, induce severe hypertension. Molecular mechanisms whereby VEGF inhibitors cause hypertension are unclear, but nitric oxide (NO) and oxidative stress may be involved. We questioned whether reactive oxygen species (ROS), important regulators of vascular function in hypertension, also play a role in VEGF inhibitor-induced vascular dysfunction. Human microvascular endothelial cells (HMECs) were stimulated with vatalanib (VEGFR inhibitor) and gefitinib (EGFR inhibitor). Normotensive male SV-129 mice (8 weeks old) were treated with Vatalanib (100 mg/Kg/day) or Gefitinib (100 mg/Kg/day). Vascular reactivity was performed mesenteric arteries using wire myograph and blood pressure was measured by tail-cuff method. Phosphorylation of eNOS was assessed by immunoblotting. ROS were measured by amplex red, lucigenin and nitrotyrosine elisa. TBARS levels were measured by lipid peroxidation assay kit and catalase activity by amplex red. Nox and antioxidant enzymes mRNA was analysed by qPCR. No changes in blood pressure were observed in animals treated with vatalanib on this dose. However acetylcholine (ACh)induced vasodilatation was impaired in those mice and phosphorylation of eNOS activation site (Ser1177) was decreased, while no changes were observed after exposure of HMECs to gefitinib. Hydrogen peroxide $\left(\mathrm{H}_{2} \mathrm{O}_{2}\right)$ levels were reduced in HMECs stimulated with vatalanib and in aorta and heart from vatalanib-treated mice. This effect was followed by an increase in catalase activity and a decrease in Nox 4 mRNA expression while Nox 5 mRNA levels were increase by vatalanib. VEGF inhibition also increased peroxynitrite $\left(\mathrm{ONOO}^{-}\right)$ levels in aorta and kidney and increased plasma TBARS levels. In kidney vatalanib increased $\mathrm{H}_{2} \mathrm{O}_{2}$ and $\mathrm{O}_{2}{ }^{-}$production which was followed by a decrease in catalase activity and $\mathrm{Nrf} 2$ nuclear translocation. Finally mRNA levels of antioxidant enzymes in HMECs, kidney and heart were decreased after exposure to vatalanib. Gefitinib only increased catalase activity and $\mathrm{ONOO}^{-}$levels in heart as well as decreased Nrf2 nuclear translocation in kidney from mice. In conclusion, our data identify novel mechanisms whereby VEGFR inhibition modulates NO signalling, antioxidant defences and ROS production in tissues and endothelial cells. These molecular processes may contribute to reduced vasorelaxation and may play a role on VEGFRI-induced hypertension.

\section{THE ROLE OF THE DNA DAMAGE RESPONSE IN VASCULAR CALCIFICATION}

${ }^{1}$ Mengxi Sun*, ${ }^{1}$ Andrew M Cobb, ${ }^{1}$ Robert Hayward, ${ }^{1}$ Qiuping P Zhang, ${ }^{2}$ Melinda Duer, ${ }^{3}$ Rukshana Shroff, ${ }^{1}$ Catherine M Shanahan. ${ }^{1}$ Cardiovascular Division, King's College London, James Black Centre, London SE5 9NE, UK; ${ }^{2}$ Department of Chemistry, University of Cambridge, Lensfield Road, Cambridge CB2 1EW, UK; ${ }^{3}$ Great Ormond Street Hospital for Children NHS Foundation Trust, London, UK

\subsection{6/heartjnl-2017-311726.223}

Introduction Vascular calcification is a hallmark of vascular ageing, and associated with vascular smooth muscle cell (VSMC) death, phenotype modulation and maladaptation. However, it remains unclear how the initial stress signals link to these downstream cellular events. Emerging evidence and our in vitro data suggest that stress may drive vascular calcification through elevated levels of DNA damage, a key factor driving cellular ageing.

Methods and Results We investigated the effects of different DNA damage signalling pathway inhibitors (ATM, ATM/ATR and PARP1) on the progression of vascular calcification. Using comet assays and western blot, we found elevated levels of DNA damage in calcified VSMCs and that inducing DNA damage accelerated rates of calcification. Chemical inhibition or siRNA knockdown of ATM, ATM/ATR or PARP signalling reduced or delayed calcification and prevented cells undergoing calcification-associated phenotype changes including osteo/chondrogenic differentiation. Prevention was associated with down-regulation of senescence and inflammatory markers suggesting the senescence associated secretory phenotype (SASP) acts to potentiate VSMC calcification.

Conclusion Taken together, these in vitro data suggest DNA damage signalling is involved in the pathological regulation of calcification. Therefore, interventions that reduce DNA damage, promote DNA damage repair, or modulate DNA damage 
signalling could be potential therapies for the prevention of vascular calcification.

\section{THE IMPACT OF NADPH OXIDASE 2 INHIBITION ON SKELETAL MUSCLE PATHOPHYSIOLOGY OF ATHEROSCLEROTIC MICE}

\begin{abstract}
${ }^{1}$ Pagona Sfyri ${ }^{*},{ }^{2}$ Nadira Y. Yuldasheva, ${ }^{3}$ Anastasia Tzimou, ${ }^{3}$ Vassilis Mougios, ${ }^{2}$ Mark Kearney, ${ }^{1}$ Antonios Matsakas. 'Molecular Physiology Laboratory, Centre for Atherothrombotic and Metabolic Disease, Hull York Medical School; ' ${ }^{2}$ Leeds Institute of Genetics Health and Therapeutics, University of Leeds; ${ }^{3}$ School of Physical Education and Sports Science, Aristotle University of Thessaloniki
\end{abstract}

\subsection{6/heartjnl-2017-311726.224}

Introduction Apolipoprotein E-deficient mice $\left(\mathrm{ApoE}^{-/}\right)$develop severe hyperlipidaemia and atherosclerotic lesions throughout the aortic root, features that are aggravated by atherogenic diets. NADPH oxidase 2 (Nox2) is an important enzymatic source of reactive oxygen species that contributes to systemic atherosclerosis in $\mathrm{ApoE}^{-/-}$mice. The aim of this study was to investigate the role of Nox 2 inhibition in skeletal muscle pathophysiology and cellular oxidative stress of $\mathrm{ApoE}^{-/-}$mice administered a Western-type of diet (WD).

Methods $\mathrm{ApoE}^{-/-}$mice were maintained on either a chow or a Western diet for 12 weeks and were treated with the Nox2dstat inhibitor or control peptide for the last 8 weeks of feeding. Skeletal muscles and the liver were dissected for molecular, biochemical and histological analysis.

Results Individual muscle fibres from $\mathrm{ApoE}^{-/-}$mice were significantly enlarged due to ectopic fat accumulation. There was an increase in hepatic inflammation and lipid deposition in response to WD administration. Importantly, there was perturbed gene expression for fatty acid metabolism and antioxidant genes, followed by evidence of oxidative stress, as shown by elevated lipid peroxidation and oxidative protein modifications such as carbonylation and tyrosine nitration in the skeletal muscle of WD-fed mice. Pharmacological inhibition of Nox2 decreased superoxide production and protein carbonylation, one of the most harmful protein modifications, in the muscle of $\mathrm{ApoE}^{-/-}$mice but had no effect on the liver.

Conclusions Our data indicate that ApoE deficiency induces oxidative damage in skeletal muscle and hepatic steatosis that are more profound under Western diet. Nox2 inhibition attenuates oxidative stress in skeletal muscle and holds promise for counteracting the impact of peripheral atherosclerosis in skeletal muscle. This study provides key evidence to better understand the pathophysiology of skeletal muscle in peripheral atherosclerosis and arterial disease; it also identifies alternative therapies to combat muscle oxidative stress.

\section{INFARCT SIZE IN A RAT MODEL OF ACUTE MYOCARDIAL INFARCTION IS REDUCED BY INTERLEUKIN-6 TRANS-SIGNALLING BLOCKADE USING SGP130FC BUT NOT AN ANTI-IL-6R MONOCLONAL ANTIBODY}

Marc Jonathan George*, Daniel Stuckey, Valerie Taylor, Aroon Hingorani, Derek Gilroy. University College London

\subsection{6/heartjnl-2017-311726.225}

Introduction Interleukin-6 (IL-6) is elevated during acute myocardial infarction (AMI) particularly after reperfusion with primary percutaneous coronary intervention (PPCI). Higher circulating levels of IL-6 and its soluble receptor (sIL-6R) are associated with adverse outcomes post AMI. Therefore while IL-6 is a potential therapeutic target in AMI, animal models employing monoclonal antibodies (MAb) against the IL-6R have failed to demonstrate benefit. We hypothesised that blockade of the pro-inflammatory aspects of IL-6 signalling (trans-signalling) with the sgp $130 \mathrm{Fc}$ protein in an animal model of AMI would result in reduced infarct size (IS) whereas blockade with a MAb against IL-6R (which blocks both the pro and anti-inflammatory actions of IL-6) would not.

Methods AMI was induced in male Sprague-Dawley rats by occluding the left-anterior descending artery for 50 minutes prior to reperfusion (analogous to PPCI). The model was characterised by measuring the temporal profile of IL-6, sIL$6 \mathrm{R}$ and other inflammatory mediators (MCP-1, KC/GRO, IL$1 \beta, \mathrm{TNF} \alpha$ ) within the heart tissue and plasma by ELISA at 2, 4, 24, 72, 120 and 168 hours post AMI (n=3-4/group). In addition, infarct progression over time (measured histologically with TTC and Evans Blue dyes and with plasma myoglobin), and leukocyte infiltration (flow-cytometry of cells obtained from heart digests) were measured. In therapeutic experiments rats received either $4 \mu \mathrm{g} / \mathrm{g}$ of a MAb against IL-6R (clone 15A7), $0.5 \mu \mathrm{g} / \mathrm{g}$ of $\mathrm{sgp} 130 \mathrm{Fc}$ or vehicle alone given intravenously 1 minute prior to reperfusion ( $n=7-8 /$ group).

Results IS/Area at risk (AAR) increased from $31.81 \%$ at 4 hours to $46.1 \%$ at 24 hours $(p=0.03)$, with no further change at 48 hours. Myoglobin peaked at 24 hours. IL-6 levels in the heart were biphasic; a robust early peak at 24 hours was followed by a trough at 24 hours, and a more sustained peak between days 3-5. Only the early peak was associated with significantly elevated circulating IL-6. The early peak was temporally associated with infarct progression and neutrophil influx, whereas the second was associated with classical mononcyte infiltration. Other inflammatory mediators followed a similar but less pronounced biphasic pattern. Cardiac and plasma sIL-6R peaked at 24 hours, coinciding with maximal cardiac neutrophil numbers. Based on these data the effect of IL-6 antagonism was assessed at 24 hours. IS/AAR after blockade with anti-IL-6R MAb was unchanged compared with control $(46.8 \%$ vs $46.1 \%)$. However, blockade with sgp130Fc resulted in a substantial reduction in IS/ARR (26.32\%, p 0.0004).

Conclusions IL-6 trans-signalling blockade with sgp130Fc but not blockade with an anti-IL-6R MAb reduces IS/AAR in an animal model of AMI with reperfusion. Ongoing experiments seek to understand the mechanisms underpinning this observation and to explore the effects on infarct healing and remodelling.

\section{BRAF INHIBITORS, SB590885 AND DABRAFENIB, ENHANCE ERK1/2 SIGNALLING IN CARDIOMYOCYTES AND PROMOTE CARDIAC HYPERTROPHY}

Daniel Meijles*, Michelle Hardyman, Kerry Rostron, Stephen Fuller, Peter Sugden, Angela Clerk. University of Reading

\subsection{6/heartjnl-2017-311726.226}

Purpose ERK1/2 are phosphorylated and activated by MKK1/2 that are phosphorylated and activated by Raf kinases. ERK1/2 promote cardiomyocyte hypertrophy and is protective, whereas 